\title{
Development and Applications of Three-dimensional Gamma Ray Tomography System Using Ray Casting Volume Rendering Techniques
}

\author{
J. M. de Oliveira Jr. ${ }^{1,2}$, F. Z. C. de Lima ${ }^{1}$, J. A. de Milito ${ }^{2}$, and A. C. G. Martins ${ }^{3}$ \\ ${ }^{1}$ Universidade de Sorocaba - UNISO, Cidade Universitária, C.P. 578, Sorocaba, SP, 18023-000, Brazil \\ ${ }^{2}$ Faculdade de Engenharia de Sorocaba - FACENS, Rod. Sen. José Ermirio de Moraes, \\ Km 1.5, Alto da Boa Vista, Sorocaba, SP, 18087-090, Brazil and \\ ${ }^{3}$ Universidade Estadual Paulista Julio de Mesquita Filho - Unesp, LAPI, \\ Av. 3 de Março, 511, Alto da Boa Vista, Sorocaba, SP, 18087-180, Brazil
}

Received on 21 July, 2005

\begin{abstract}
Computed Tomography (CT) has made possible visualization of physical structures in the interior of an object in their real relative spatial position and orientation. Another important contribution of CT is to greatly improve abilities to distinguish regions with different gamma ray transmittance and to separate over-lying structures. The mathematical problem of CT imaging is that of estimating an image from its projections. In this work, the experimental setup was performed using the Mini Computerized Tomograph of Uniso (MTCU). This tomograph system operates with a gamma ray source of ${ }^{241} \mathrm{Am}$ (photons of $60 \mathrm{KeV}$ and $100 \mathrm{mCi}$ of intensity) and a $\mathrm{NaI}(\mathrm{Tl})$ solid state detector. The system features translation and rotation scanning modes, a $100 \mathrm{~mm}$ effective field of view, $1 \mathrm{~mm}$ of spatial resolution and $5 \%$ to $10 \%$ of density resolution. The image reconstruction problem can be solved using two different algorithms: Algebraic Reconstruction Techniques (ART) or Discrete Filtered Backprojection (FBP). The three-dimensional image reconstruction method discussed in this work, involves obtaining two-dimensional (2D) gamma ray tomography images and then combining these images into a threedimensional (3D) volume data. An opacity, shading and color is attributed to each volume element (voxel) and the resulting scene is projected in a picture plane to be display in a monitor. Our implementation was developed to be used with MTCU data and was based in the Ray casting volume rendering technique. The use of such technique for nondestructive evaluation is a powerful tool to enable a visual trip inside an object without physically opening or cutting it. Experimental and theoretical methods used are discussed and results of experiments using the $3 \mathrm{D}$ reconstruction techniques are presented. A particular use of this technique to study concrete properties, such as stones distributions, visualization of structural occurrences inside concrete samples is discussed.
\end{abstract}

\section{INTRODUCTION}

Computerized Tomography (TC) has made possible visualization of physical structures in the interior of an object without physically opening or cutting it $[1,2]$. Though two dimensional (2D) gamma ray tomography has been used in many areas [3-6] as in important tool of nondestructive evaluation, its combination with volume visualization technique [7-10], which gives a three-dimensional (3D) tomographic image, can be a very powerful tool to analyze objects without destructing it. In order to explore this technique, the 2D gamma ray computed tomography system of Sorocaba University (MTCU) [11] was modified, so that slices of an object in different positions could be acquire, allowing 3D reconstruction. Volume rendering techniques were used for the visualization of acquire volume data. Applications of these techniques, such as oppening the object layer by layer, cutting it at any particular angle and rotation are presented using a concrete sample as the studied object. The detection, quantification and further analysis of structural changes in cementbased material with $3 \mathrm{D}$ visualization offers the potential for more rational approach to design, testing, repair, or replacement of concrete structures [12-14]. An overview on the applications of non destructive testing techniques for assessment of concrete structures is given by Baladev Raj and Jayakumar [15]. This article discusses the theoretical method and experi- mental setup for obtaining 2D tomograms of concrete samples and then the use of these data to performed the 3D reconstruction. In order to study the shape and spatial distribution of coarse aggregate, voids and cracks distribution, we have developed a 3D visualization kit denominate "TOMOGRAFIA TRI-DIMENSIONAL - TTD", based on direct volume rendering. This package runs on PC, using the Windows platform.

\section{GAMMA RAY TOMOGRAPHY: MATERIALS AND METHODS}

The gamma ray CT miniscanner employed in this experiment was built at the Experimental Nuclear Physics Laboratory at University of Sorocaba. This scanner allows the use of nondestructive techniques for visualizing features in the interior of opaque solid objects to obtain digital information on their 3D geometry and topology. In the case of concrete, the aggregates used can be visualized inside a samples. The images to be shown in this paper were all taken with MTCU. The tomograph operates with a gamma ray source of ${ }^{241} \mathrm{Am}$ (photons of $60 \mathrm{KeV}$ and $100 \mathrm{mCi}$ of intensity) and a $\mathrm{NaI}(\mathrm{Tl})$ solid state detector. A PC controls the data acquisition system as well as the movement of translation and rotation of the sample. A tomographic image can be reconstructed, after a number of profiles of narrow-beam transmission are made at different orientations. These measurements represent the to- 


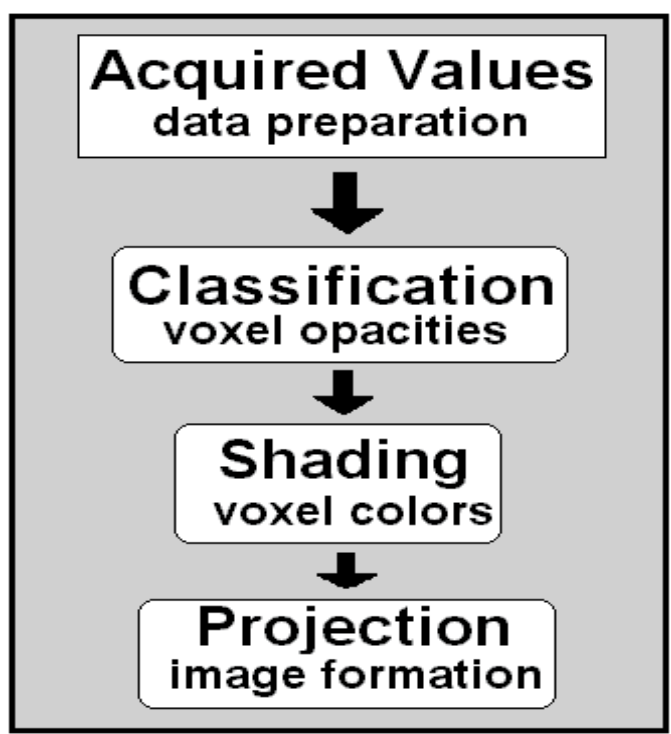

FIG. 1: Esquematic view of rendering pipeline.

tal linear attenuation coefficient of $\gamma$-rays along the path of the ray. The image reconstruction problem can be solved, in our case, using two different algorithms: i) Algebraic Reconstruction Techniques (ART) [16] and ii) Discrete Filtered Backprojection (FBP) $[17,18]$. Visual criterion were used to choice which algorithm produce the best image. The results is a tomographic 2D image of a cross section of the specimen studied. With several 2D images, a stacking procedure is employed to produce the $3 \mathrm{D}$ volume data. The concrete samples used in this study were made using cement type Portland-CPII, fine natural sand, stones (coarse aggregate) and water. The cylindrical concrete samples had measured between $15 \mathrm{~mm}$ to $50 \mathrm{~mm}$ of diameter and $50 \mathrm{~mm}$ of height.

\section{THEORETICAL DISCUSSIONS: VOLUME RENDERING TECHNIQUES}

Volume rendering is a family of techniques for visualizing sampled scalar or vector fields of three spatial dimensions without fitting geometric primitives to the data. The techniques used in the process of visualization can be resumed in four steps showed in Figure 1.

The first step is data preparation, which can include some interpolation between 2D acquired images, contrast enhancement and use of some filters to improve the data quality. These $2 \mathrm{D}$ data are then used as input for the formation the $3 \mathrm{D}$ volume data. The volume data are divided into volume elements called voxels. This voxel array are then used in the classification procedure, where one opacity is associated to each voxel by making use of transfer function like the one shown in Eq.(1), proposed by Levoy [10]. In this equation an opacity $\alpha_{v}$ is associate to all voxels having selected value of color or intensity $f_{v}$, and assigning an opacity zero to all other voxel. To avoid aliasing artifacts, we would also like voxel having values close to $f_{v}$ to be assigned opacities close to $\alpha_{v}$.

$$
\alpha\left(x_{i}\right)=\alpha_{v} \begin{cases}1 & \text { if }\left|\nabla f\left(x_{i}\right)\right|=0 \\ & \text { and } f\left(x_{i}\right)=f_{v} \\ 1-\frac{1}{r}\left|\frac{\left(f_{v}-f\left(x_{i}\right)\right.}{\left|\nabla f\left(x_{i}\right)\right|}\right| & \text { if }\left|\nabla f\left(x_{i}\right)\right|>0 \\ & \text { and } f\left(x_{i}\right)-r \\ & \left|\nabla f\left(x_{i}\right)\right| \leq f_{v} \leq \\ & f\left(x_{i}\right)+r\left|\nabla f\left(x_{i}\right)\right| \\ 0 & \text { otherwise }\end{cases}
$$

In Eq. (1) $r$ is a voxel thickness and $\left|\nabla f\left(x_{i}\right)\right|$ is the gradient vector. This transfer function enables to view some special structures contained within the volume data. In a separate step, the voxel array is used as input to a shading model like that showed in Eq. (2), proposed by Phong [19, 20].

$$
\begin{aligned}
& C_{\lambda}\left(x_{i}\right)=C_{\lambda}\left(x_{i}\right) K_{a}+ \\
& +\frac{C_{\lambda}\left(x_{i}\right)\left\{K_{d}\left[N\left(x_{i}\right) \cdot L\right]+K_{S}\left[N\left(x_{i}\right) \cdot H\right]^{n}\right\}}{d\left(x_{i}\right)}
\end{aligned}
$$

where $C_{\lambda}\left(x_{i}\right)$ is the component of color at voxel location $x_{i}$, $K_{a}, K_{d}$ and $K_{s}$ are respectively the ambient, diffuse and specular reflection coeficient of light, $n$ is the exponent used to approximate highlight, $d\left(x_{i}\right)$ is the perpendicular distance from picture plane to voxel location, $N\left(x_{i}\right)$ is the surface normal vector at voxel location, $L$ is the normalized vector in the direction of light source and $H$ is the normalized vector in the direction of maximum highlight. The shaded color volume provides a satisfactory illusion of smooth and 3D shape cues. This step simulate the sum of the light emitted by the volume and scattered by the surfaces. Finally rays are then cast into these two arrays from the observer eyepoint. For each ray a vector of sample colors and opacities is computed by resampling the voxel database at some evenly spaced locations along the ray. Eq. (3) shows the ray casting method used to perform these projections.

$$
I(u, v)=\sum_{i=1}^{k}\left[C_{\lambda}\left(x_{i}\right)_{i} \alpha\left(x_{i}\right)_{i} \prod_{j=i+1}^{k}\left(1-\alpha\left(x_{i}\right)_{j}\right)\right]
$$

where $I(u, v)$ is the final color at pixel location $(u, v)$. The final image can be showed in different angles and cut planes can be introduced to view selected portion of the volume. These effects were implemented in our program, before of the rays casting. In our implementation we cast only one ray per pixel.

\section{EXPERIMENTAL RESULTS AND DISCUSSION}

Several sets of samples were especially fabricated to test the $2 \mathrm{D}$ and 3D algorithm. Figure 2 shows a 3D volume rendered image of concrete sample by combining 81 2D CT scan images into a volume data. 


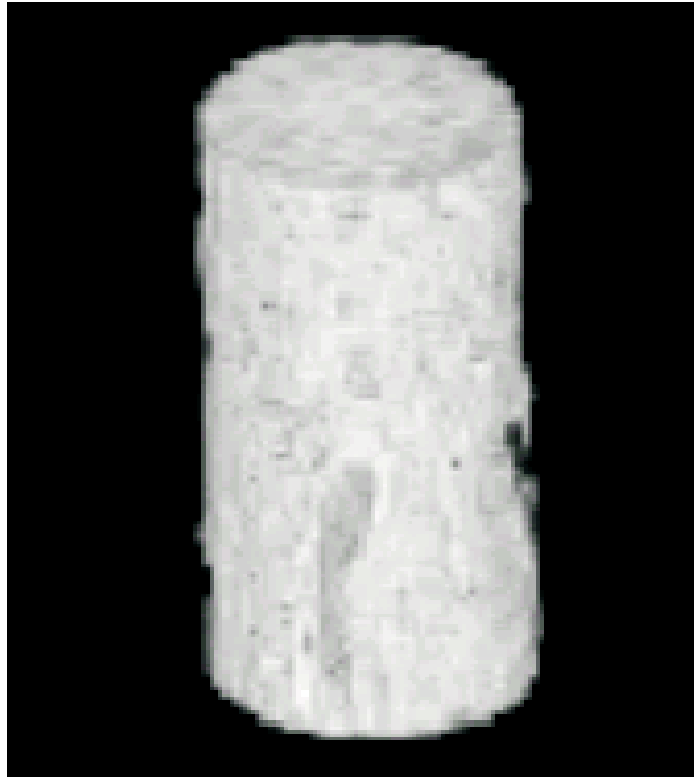

FIG. 2: The Three-dimensional rendering of a concrete specimem, stacking 81 cross-sectional images.

Each 2 D image contais 81 per 81 pixel, totalizing $81^{3}$ voxels. The resolution of the image is $1 \mathrm{~mm} /$ pixel. Figure 3 shows the distribution of coarse aggregates inside the sample. This image was obtained using Levoy's transfer function to selected only the interest part of the concrete (coarse aggregate).

Figure 4 shows how the application of the tools implemented in the TTD program can produce some especial views of the object in study. The upper and lower parts of the figure shows one view obtained using rotation tools associated with some virtual cutting planes. Both figures were produced using Levoy's transfer function.

Another interesting feature of the implemented tools, is the possibility to use them to make a Stereology study [21, 22] of the concrete samples, i. e., obtaining the statistical information of geometric quantities of a three-dimensional sample from observations made on two-dimensional section. We showed in the upper part of figure 5 the fotography, reconstructed tomography image of a concrete sample and some possible three orthogonal cut planes used in Stereology study, respectively. In the lower part of figure 5 we showed these orthogonal cut planes, obtained virtualy, where it is possible to observe some damage in the concrete, like the big crack extension and some air voids. In concrete, cracks are thought to originate from one or more porous voids, and they may even spread preferentially through voids and pre-existing cracks. We hope that the detailed mechanisms of crack origination and propagation can be better studied using the $3 \mathrm{D}$ gamma rays imaging and the tools implanted in program TTD.

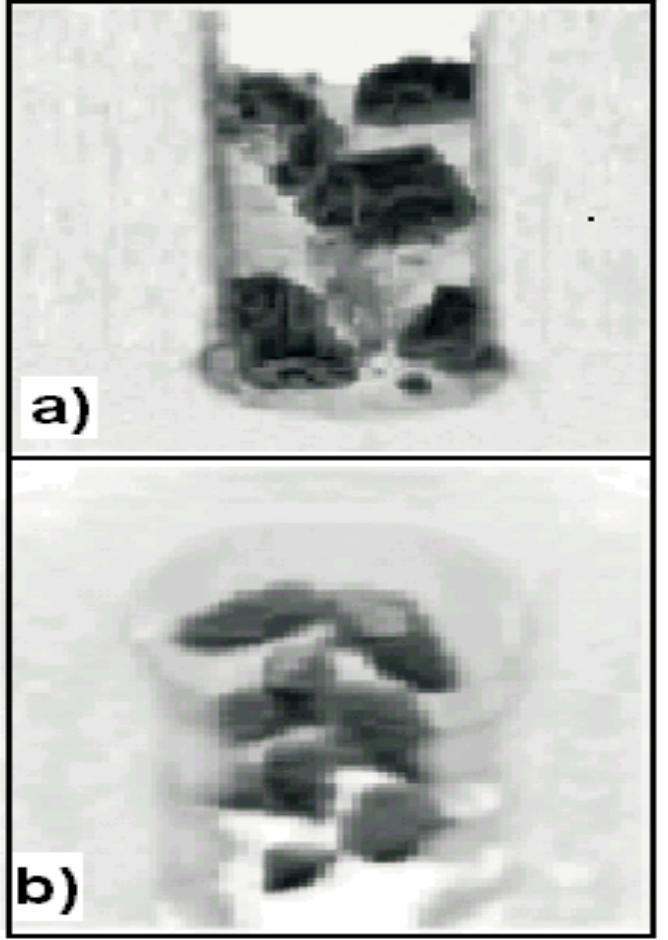

FIG. 3: Images of two concrete block with $81^{3}$ voxels. Coarse Aggregates (high density) in figures a) and b) appear in black, while the gray portion of figure represents air around the concrete block. Mortar was removed using Levoy's transfer function. In these two figures some viewing cutting planes were introduced and a rotation was performed to generate a better view.

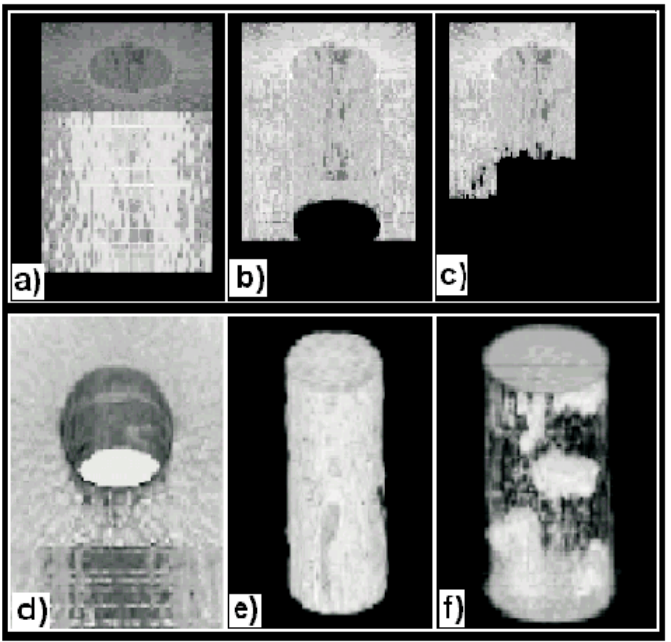

FIG. 4: The figure shows a miscellaneous tools, implanted in the TTD program. These tools can be used to produce some especial views in the object in study. 


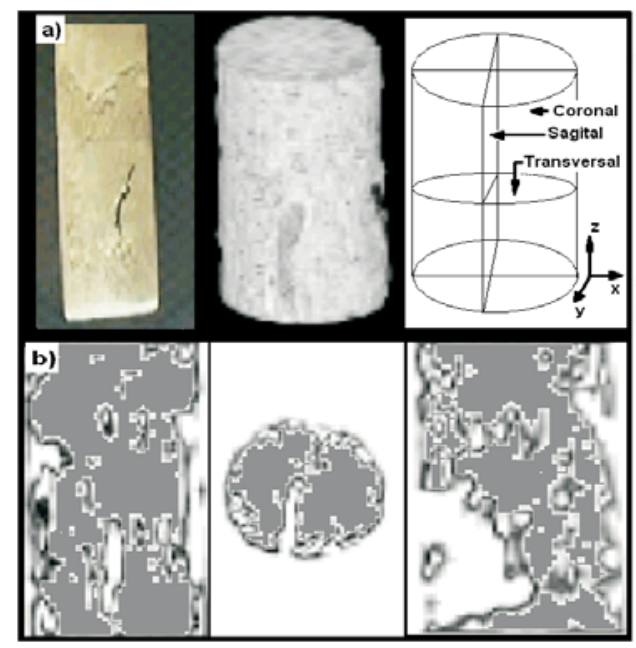

FIG. 5: (a) fotography of a concrete sample, reconstructed tomography image and three orthogonal cut planes respectively, (b) three orthogonal images (coronal, transverse and sagital) obtained from virtual cutting tools implemented in the TTD program.

\section{CONCLUSION}

There are a number of industrial applications where the technique of three- dimensional gamma ray tomography can be very useful. We showed one example of application applied in qualitative concrete analysis, where we showed that it is possible to perform the identification of some structures and imperfection in the concrete, such as stone distributions, air voids as well as cracks distribution and extension. The results of application of the tools implemented in TTD program, confirm the high potential of the method for concrete analysis and open possibilities to use this techniques in others areas.

\section{Acknowledgments}

The authors would like too thank Mr. Dirk Bekel and Mr. Rubens Rehder directors of linear product line of SCHAEFFLER BRASILl LTDA industry, for the donation of a linear guide used in the construction of a Mini Computerized Tomograph of UNISO and Fundação de Amparo à Pesquisa do Estado de São Paulo - FAPESP, for financial support.
[1] G. N. Hounsfield, British Journal of Radiology, 46, 1016 (1973).

[2] J. Ambrose, British Journal of Radiology, 46, 1023 (1973).

[3] J. M. Oliveira Jr. A. C. G. Martins, and J. A. DE Milito, Braz. Jour. Phys. 34, 1020 (2004)

[4] P. E. Cruvinel, R. Cesareo, S. Crestana, and S. Mascarenhas, IEEE Trans. Instrum. Meas., 39(5), 745 (1990).

[5] D. Braz, L. M. G. Motta, R. T. Lopes, Appl. Rad. and Isot., 50, 661 (1999).

[6] C. R. Appoloni, A. C. Nardocci, and M. M. Obutti, Ciência e Cultura, 40, 387 (1987).

[7] M. P. Baker, D. Hearn, Computer Graphics-Version C,2 Ed. New Jersey: Prentice-Hall (1997).

[8] A. E. Kaufman, Volume Graphics Computer, 26(7), 51 (1993).

[9] R. A. Derbin, L. Carpenter, and P. Hanharan, Computer Graphics, 2265 (1988).

[10] M. Levoy, IEEE Computer Graphics \& Applications, 8, 29 (1988).

[11] J. M. Oliveira Jr., Braz. Jour. Phys. 33, 273 (2003)

[12] L. B. Wang, J. D. Frost, G. Z. Voyiadjis, T. P. Harman, Mecha- nics of Materials, 35, 777 (2003)

[13] E. J. Garboczi, Cement and Concrete Research 32, 1621 (2002)

[14] S. R. Stock, N. K. Naik, A. P. Wilkinson, and K. E. Kurtis, Cement and Concrete Research 32, 1673 (2002)

[15] B. Raj and T. Jayakumar, Journal of Non Destructive Evaluation, 3 29, (2001).

[16] R. Gordon, R. Bender, and G. T. Herman, J. Theor. Biol. 29, 471 (1970).

[17] R. N. Bracewell and A. C. Riddle, Astrophys. J. 150, 427 (1967).

[18] G. N. Ramachandran and A. V. Lakshminarayanan, Proc. Nat. Acad. Sci. USA 68, 2236 (1971).

[19] B. T. Phong, Communications of the ACM, 18, 311 (1975).

[20] J. Blinn, Computer Graphics, 11, 192 (1977).

[21] R. T. DeHoff, F. R. Rhines, Quantitative microscopy. McGrawHill, New-York (1968).

[22] E. E. Underwood. Quantitative Stereology. Addison-Wesley, Reading (1970) 